\title{
Comparison of Renal Artery vs Renal Artery-Vein Clamping During Partial Nephrectomy: A System Review and Meta-Analysis
}

\author{
Jian Cao, MD, PhD, Shuai Zhu, MS, Mingji Ye, MD, Kan Liu, MD, Zhizhong Liu, MD, PhD, \\ Weiqing Han, MD, and Yu Xie, MD, PhD
}

\begin{abstract}
Background: Although artery-only (AO) clamping has been proposed to minimize ischemic renal damage compared with artery-vein (AV) clamping, the benefit of AO clamping during laparoscopic partial nephrectomy (LPN) is still controversial. We performed a systematic review and meta-analysis to test the difference between AO clamping and AV clamping in partial nephrectomy (PN).

Materials and Methods: A systematic review of the literature on PubMed, Web of Science, the Cochrane Library, and Embase was conducted according to the Preferred Reporting Items for Systematic Reviews and Meta-Analyses Statement to search-related studies. Data were extracted using a reporting checklist proposed by the Meta-analysis of Observational Studies in Epidemiology Group. RevMan 5.3 software and Stata 12.0 were used to do meta-analysis.

Results: The present meta-analysis included 2 retrospective and 3 prospective studies, including 242 patients who underwent AO clamping and 369 patients who underwent AV clamping, which compared AO and AV clamping in LPN for renal cell carcinoma. At baseline, no statistically significant differences were detected between $\mathrm{AO}$ and $\mathrm{AV}$ clamping groups in terms of body mass index $(p=0.23)$, tumor size $(p=0.95)$, but $\mathrm{AO}$ clamping group had significantly lower RENAL Score (fixed effects [FE]: weighted mean difference [WMD]: $0.36, p=0.007)$. For surgical outcomes analysis, no significant difference was detected regarding to warm ischemia $(p=0.58)$, operating time $(p=0.40)$, transfusion rate $(p=0.58)$, and estimated blood loss $(p=0.35)$ between two groups. The assessment of renal function by creatinine value both at the early postoperative $(p=0.36)$ and at last follow-up $(p=0.38)$ revealed no difference. There was no significant difference in estimated glomerular filtration rate (eGFR) $(p=0.62)$, and at the early postoperative percentage decrease of eGFR $(p=0.79)$. However, a higher percentage decrease of eGFR decrease at last follow-up was demonstrated for the AV clamping group (FE: WMD: $2.42, p<0.00001$ ).

Conclusion: These results suggest that AO clamping might be a better choice for PN in long term. Randomized controlled trial studies with larger sample numbers and long-term follow-up and split renal function assessment should be conducted in the future to confirm our conclusion.
\end{abstract}

Keywords: renal artery clamping, renal artery-vein clamping, partial nephrectomy

\section{Introduction}

$\mathbf{P}$ ARTIAL NEPHRECTOMY (PN) has been gaining popularity in the last decade and become the gold standard treatment for patients with small renal tumors. ${ }^{1}$ Renal artery clamping techniques is a key point in PN for renal function preservation, and renal artery with or without renal vein clamping during $\mathrm{PN}$ is one of these techniques. Theoretically, artery-only (AO) clamping may minimize ischemic renal damage compared with artery-vein (AV) clamping because of retrograde venous perfusion during AO clamping. On the contrary, AV clamping blocks venous backflow during tumorectomy, allowing better visualization of the renal tumor margins control and subsequent renal reconstruction. ${ }^{2-4}$

Several animal studies have shown that AO clamping resulted in increased tolerance to ischemic damage compared with AV clamping during PN. ${ }^{3-5}$ However, most of recent studies among human subjects undergoing PN show no 
benefit of retrograde venous perfusion associated with $\mathrm{AO} v s$ AV clamping during $\mathrm{PN}$. $^{2,3,6,7}$ However, a retrospective study by Gong suggested that AO occlusion may provide superior renal preservation during laparoscopic partial nephrectomy (LPN). ${ }^{8}$ These studies yield inconsistent results and thus fail to provide a general consensus on the renoprotective effect of AO vs AV clamping during PN. In an effort to elucidate any surgical and renal function benefit from AO vs AV clamping, we conducted a systematic review and meta-analysis of the available literature to compare AO vs AV clamping on the clinical characteristics and surgical and functional outcomes of renal cell carcinoma (RCC) patients who underwent PN.

\section{Materials and Methods}

\section{Literature search strategy}

Systematic literature searches of PubMed (through May 2019), EMBASE (through May 2019), Web of Science (1950 to May 2019), and the Cochrane Library (through May 2019) were conducted to retrieve potentially relevant studies, according to the Preferred Reporting Items for Systematic Reviews and Meta-Analysis (PRISMA) statement guidelines, ${ }^{9}$ We used the keywords as follows: ("partial nephrectomy" OR "nephron sparing surgery") AND ("renal artery only clamping" OR "renal artery and vein clamping") AND ("comparative studies"). No restriction was applied. We also manually searched the references of related articles for additional studies. Two reviewers conducted the searching independently, and all divergences were diminished through group discussion, and consensuses were reached.

\section{Inclusion criteria and exclusion criteria}

Two authors reviewed all candidate studies (J.C. and S.Z.), and divergences were solved by focus groups. Studies fulfilling the following criteria were selected for the metaanalysis: patients who were diagnosed with RCC and received $\mathrm{PN}$ treatment; the study that directly compared $\mathrm{AO}$ vs AV clamping; and sufficient original data for comparison could be extracted or calculated. We excluded noncomparable studies, such as reviews, letters, case reports, and nonhuman studies; studies without sufficient data to analyze AO and AV clamping; and studies not in English language were excluded as well.

\section{Data extraction}

The reporting checklist proposed by the Meta-analysis of Observational Studies in Epidemiology (MOOSE) Group was applied to extracted related data. ${ }^{10}$ Data extraction was performed independently by two authors, and any discrepancies were resolved by group discussion. The first author, publication year, country, study design, age of patients, patients numbers, and baseline clinical characteristics were extracted from related studies. The main surgical and functional parameters of interest included warm ischemia time, operating time (OT), estimated blood loss (EBL), transfusion rate (TR), tumor size (TS), RENAL Score, creatinine, postoperative estimated glomerular filtration rate (eGFR), and percentage decrease in baseline eGFR (\% eGFR).

\section{Statistical analysis}

Inverse variance test was used to pool weighted mean differences (WMDs) for continuous variables, and dichotomous variables were pooled as odds ratios and $95 \%$ confidence intervals (95\% CIs) using Mantel-Haenszel (M-H) test. The Cochrane Q statistic and $I^{2}$ statistic were applied to assess statistical heterogeneity among studies. The value of $p<0.10$ or $I^{2}>50 \%$ was used to account for substantial heterogeneity among studies. If there was no evidence of heterogeneity among studies, a fixed effects (FE) model was used; otherwise a random-effects (RE) model was used. The "leave-one-out" cross-validation procedure was applied to evaluate sensitivity among studies. Publication bias of studies was analyzed via the Begg's funnel plot and the Egger's linear regression test. Review Manager Version 5.0 (The Cochrane Collaboration, Oxford, London, UK) and STATA 12.0 software (Stata Corporation, College Station, TX) were used to conduct statistical analyses. Two-tailed $p<0.05$ was considered statistically significant.

\section{Quality assessment}

The quality assessment of included studies was conducted following the Newcastle Ottawa scale (NOS) ${ }^{11}$ for nonrandomized controlled trials (RCTs). Each study was evaluated by eight methodology items with a score ranging from 0 to 9 . Studies with scores $\leq 5$ were considered as low quality, 6-7 considered as intermediate quality, and 8-9 as high quality.

\section{Results \\ Clinical characteristics and quality assessment of eligible studies}

The flow chart of the literature search is shown in Figure 1. From a total of 337 potentially relevant abstracts initially retrieved, 197 records were removed after review of title/ abstract if there were case reports, letters, review, metaanalysis, or nonhuman studies; 30 studies were excluded because of data deficiencies and surgical technique on offclamping or artery selective clamping. At last five studies that complied with the inclusion criteria were included in the meta-analysis. ${ }^{2,6-8,12}$ The clinical characteristics of all five included studies are shown in Table 1. A total of 611 patients with RCC patients received PN were included in this metaanalysis, of whom 242 applied AO clamping and 369 used AV clamping during PN. All of these studies were non-RCTs (level of evidence $3 b$ ), revealing a high risk of selection bias for all the studies. For quality evaluated by the NOS, all included studies were of intermediate quality (score 6 for one and score 7 for four) (Table 1).

\section{Meta-analysis of clinical characteristics}

At baseline, no statistically significant differences were detected between $\mathrm{AO}$ and $\mathrm{AV}$ clamping groups in terms of body mass index (BMI) (RE: WMD: $-0.44,95 \%$ CI: $[-1.15$, $0.27], p=0.23 ; I^{2}=54 \%$ ), TS (RE: WMD: -0.01 , 95\% CI: $\left.[-0.47,0.44], p=0.95 ; I^{2}=71 \%\right)$. However, patients in the AO clamping group had significantly lower RENAL Score than the AV clamping group (FE: WMD: 0.36, 95\% CI: $[0.10,0.62], p=0.007 ; I^{2}=43 \%$ ) (Fig. 2A-C, Table 2). 


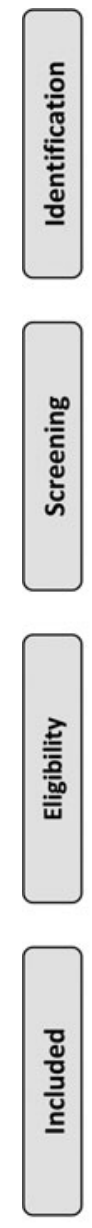

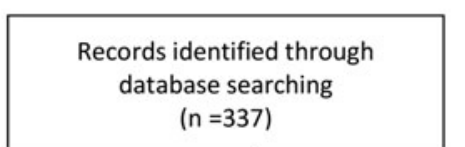

$(n=337$

\section{Meta-analysis of surgical outcomes}

Pooled analysis of surgical outcomes revealed no significant difference regarding warm ischemia (RE: WMD: 1.97, 95\% CI: $[-4.93,8.88], p=0.58 ; I^{2}=97 \%$ ), OT (FE: WMD: $3.20,95 \%$ CI: $[-4.32,10.71], p=0.40 ; I^{2}=0 \%$ ), TR (FE: weighted odds ratio: $1.34,95 \%$ CI: $[-0.48,3.78], p=0.58$; $I^{2}=0 \%$ ), and EBL (RE: WMD: 33.26, 95\% CI: [-35.88, 102.40], $p=0.35 ; I^{2}=79 \%$ ) between two groups (Fig. 3A-D, Table 2).

\section{Meta-analysis of functional outcomes}

The assessment of renal function by creatinine value both at the early postoperative (RE: WMD: $-0.18,95 \%$ CI: $[-0.56$, $0.2], p=0.36 ; I^{2}=85 \%$ ) and at last follow-up (RE: WMD: $-0.11,95 \%$ CI: $[-0.36,0.14], p=0.38 ; I^{2}=50 \%$ ) revealed no
FIG. 1. PRISMA 2009

flow diagram. Data from: Moher D, Liberati A, Tetzlaff J, Altman DG, The PRISMA Group (2009). Preferred Reporting Items for Systematic Reviews and Meta-Analyses: The PRISMA Statement. PLoS Med 6(7): e1000097. doi: 10.1371/journal .pmed1000097. For more information, visit www .prisma-statement.org. PRISMA = Preferred Reporting Items for Systematic Reviews and Meta-Analysis.

Table 1. Main Characteristics of the Studies Included in the Meta-Analysis

\begin{tabular}{|c|c|c|c|c|c|c|c|c|c|c|c|c|}
\hline \multirow{2}{*}{$\begin{array}{l}\text { First } \\
\text { author }\end{array}$} & \multirow[b]{2}{*}{ Year } & \multirow[b]{2}{*}{ Country } & \multirow{2}{*}{$\begin{array}{c}\text { Study } \\
\text { design }\end{array}$} & \multicolumn{2}{|c|}{ Age (year) } & \multicolumn{2}{|c|}{ Sample size (n) } & \multicolumn{2}{|c|}{ Tumor size $(\mathrm{cm})$} & \multicolumn{2}{|c|}{ RENAL Score } & \multirow{2}{*}{$\begin{array}{l}\text { NOS } \\
\text { Score }\end{array}$} \\
\hline & & & & $A O$ & $A V$ & $A O$ & $A V$ & $A O$ & $A V$ & $A O$ & $A V$ & \\
\hline Edward & 2008 & USA & Retrospective & 63.1 & 57.9 & 25 & 53 & 2.4 & 2.9 & - & - & 6 \\
\hline Kyle & 2016 & USA & Retrospective & 61.0 & 60.0 & 70 & 163 & 2.9 & 3.0 & 6.5 & 6.0 & 6 \\
\hline Zhuo-Wei & 2013 & USA & Prospective & 58.1 & 58.0 & 12 & 25 & 4.6 & 3.6 & 8.0 & 8.0 & 7 \\
\hline Annie & 2012 & USA & Prospective & 59.9 & 59.4 & 103 & 102 & 3.2 & 3.4 & - & - & 7 \\
\hline Yasuhito & 2013 & Japan & Prospective & 63.6 & 60.1 & 32 & 26 & 2.8 & 3.0 & 5.5 & 5.6 & 7 \\
\hline
\end{tabular}

$\mathrm{AV}=$ artery-vein $; \mathrm{AO}=$ artery-only $; \mathrm{NOS}=$ Newcastle Ottawa scale. 


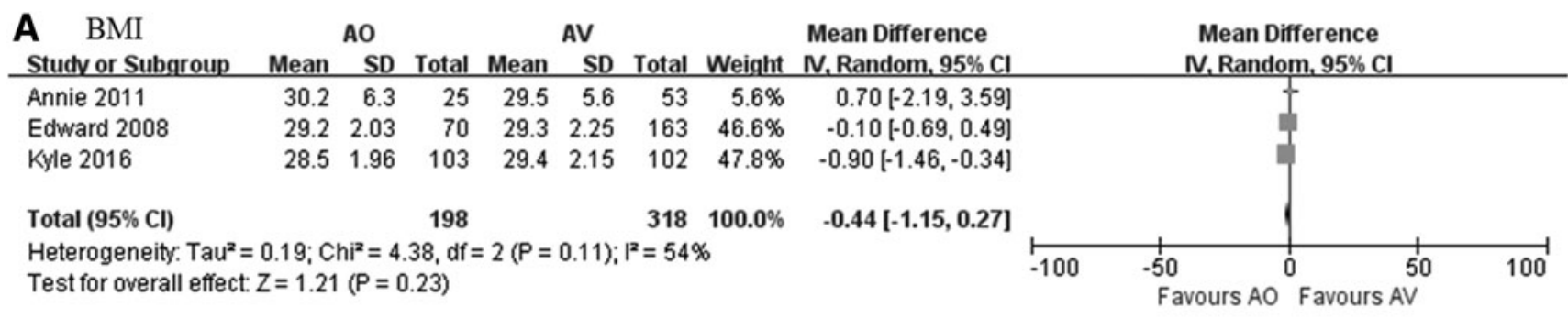

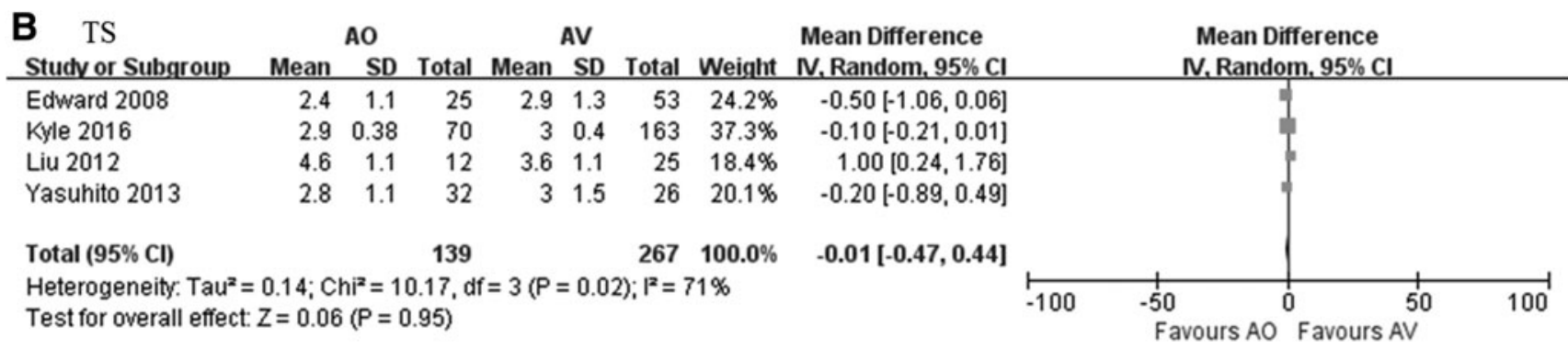

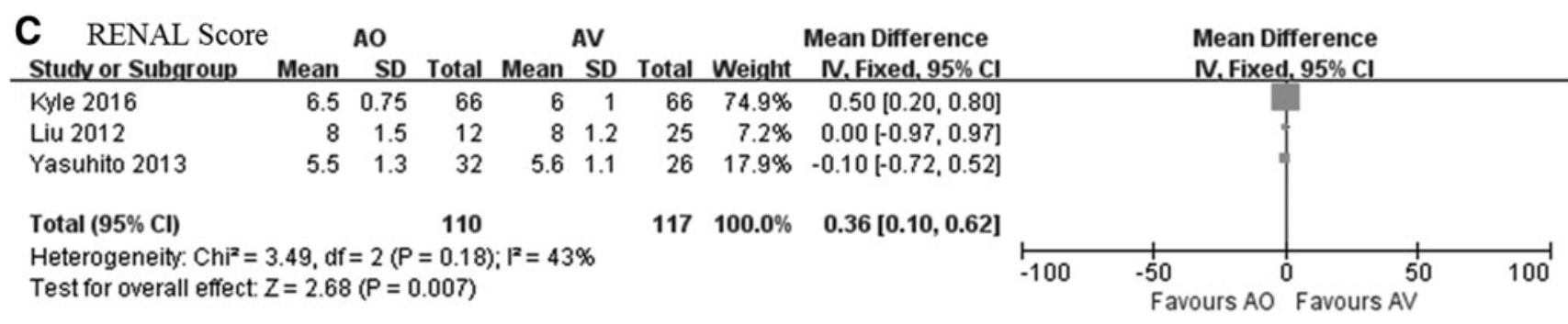

FIG. 2. Forest plots of clinical characteristics. (A) BMI: (B) TS: (C) RENAL Score.

were reliable and robust (Supplementary Fig. S2). The Begg funnel plot and Egger funnel plot showed no statistically significant publication bias in outcomes of interest (Table 2).

\section{Discussion}

As the population of patients growing with older age, hypertension, or diabetes, $\mathrm{PN}$ with better surgical and functional recovery would become a more common choice for patients of RCC. However, WI injury during renal occlusion limited the use of PN. One proposed way to reduce the detrimental effect of WI is renal AO clamping during PN, which has been demonstrated effectively in animal models. Colli et al. compared the ischemic damage of $\mathrm{AO} v \mathrm{AV}$ clamping in two animal models, and they demonstrated that oxygen saturation was picking up two times faster, and mean postclamp 8isoprostane level, which was a biomarker to quantitate renal reperfusion injury, was a 1.54-fold decrease with clamping renal artery alone compared to clamping artery/vein together. ${ }^{3}$ Orvieto and colleagues evaluated the impact of laparoscopic $v s$ open surgery on the recovery of renal function

Table 2. Summary of all the Meta-Analysis and Publication Bias

\begin{tabular}{|c|c|c|c|c|c|c|c|c|}
\hline \multirow[b]{2}{*}{ Analysis } & \multirow{2}{*}{$\begin{array}{l}\text { No. of } \\
\text { studies }\end{array}$} & \multirow{2}{*}{$\begin{array}{l}\text { Effect } \\
\text { model }\end{array}$} & \multicolumn{2}{|c|}{ Study heterogeneity } & \multirow{2}{*}{$\begin{array}{c}\text { Pooled WMD/OR } \\
{[95 \% \mathrm{CI}]}\end{array}$} & \multirow[b]{2}{*}{$\mathrm{p}$} & \multicolumn{2}{|c|}{ Begg test } \\
\hline & & & $\mathrm{I}^{2}(\%)$ & $\mathrm{p}$ & & & $\mathrm{t}$ & $\mathrm{p}$ \\
\hline BMI & 3 & Random & 54 & 0.11 & $-0.44[-1.15,0.27]$ & 0.23 & 1.04 & 0.30 \\
\hline TS & 4 & Random & 71 & 0.02 & $-0.01[-0.47,0.44]$ & 0.95 & 1.02 & 0.31 \\
\hline RENAL Score & 3 & Fixed & 43 & 0.180 .36 & {$[0.10,0.62]$} & 0.007 & 0.00 & 1.00 \\
\hline WI & 5 & Random & 97 & $<0.001$ & $1.97[-4.93,8.88]$ & 0.58 & -0.24 & 1.00 \\
\hline OT & 3 & Fixed & 0 & 0.54 & $3.20[-4.32,10.71]$ & 0.40 & 0.00 & 1.00 \\
\hline TR & 2 & Fixed & 0 & 0.75 & $1.34[-0.48,3.78]$ & 0.58 & 0.00 & 1.00 \\
\hline EBL & 5 & Random & 79 & 0.0008 & $33.26[-35.88,102.40]$ & 0.35 & 0.24 & 0.81 \\
\hline E-creatinine & 2 & Random & 85 & 0.009 & $-0.18[-0.56,0.2]$ & 0.36 & 0.00 & 1.00 \\
\hline L-creatinine & 2 & Random & 50 & 0.16 & $-0.11[-0.36,0.14]$ & 0.38 & 0.00 & 1.00 \\
\hline eGFR & 2 & Fixed & 0 & 0.46 & $-1.36[-6.67,4.04]$ & 0.62 & 0.00 & 1.00 \\
\hline E-\%eGFR & 3 & Random & 91 & $<0.0001$ & $-0.40[-3.29,2.5]$ & 0.79 & 0.00 & 1.00 \\
\hline L-\%eGFR & 4 & Fixed & 0 & 0.51 & $2.42[1.66,3.18]$ & $<0.00001$ & 0.34 & 0.73 \\
\hline
\end{tabular}

$p$ value in bold represents statistical significance.

95\% $\mathrm{CI}=95 \%$ confidence interval; $\mathrm{BMI}=$ body mass index; $\mathrm{EBL}=$ estimated blood loss; eGFR = estimated glomerular filtration rate; $\mathrm{OR}=$ odds ratio; $\mathrm{OT}=$ operating time $\mathrm{TR}=$ transfusion rate; $\mathrm{TS}=$ tumor size; $\mathrm{WI}=$ warm ischemia; $\mathrm{WMD}=$ weighted mean difference . 


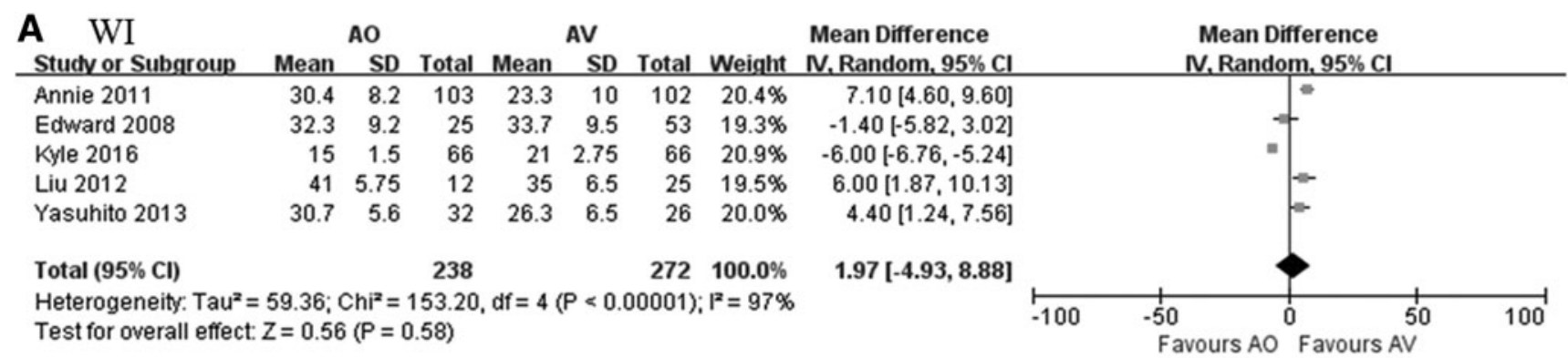

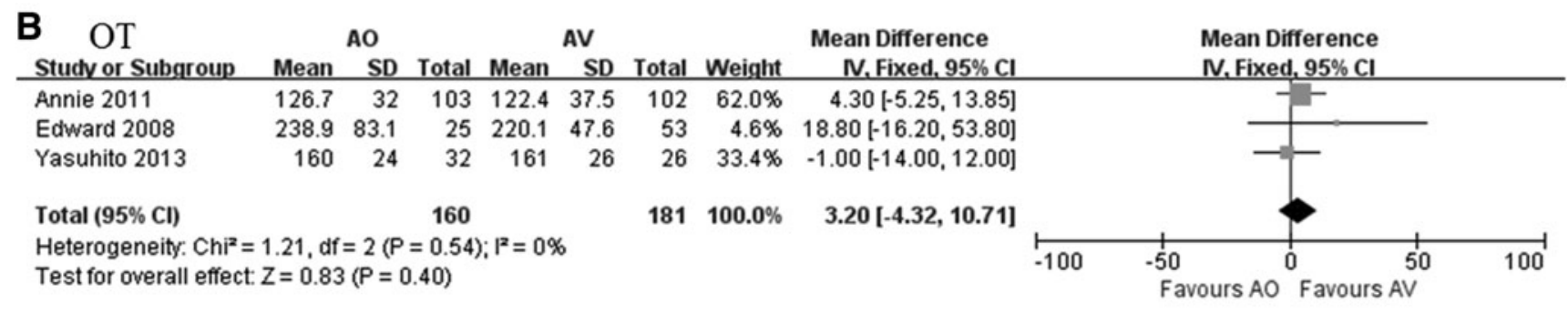

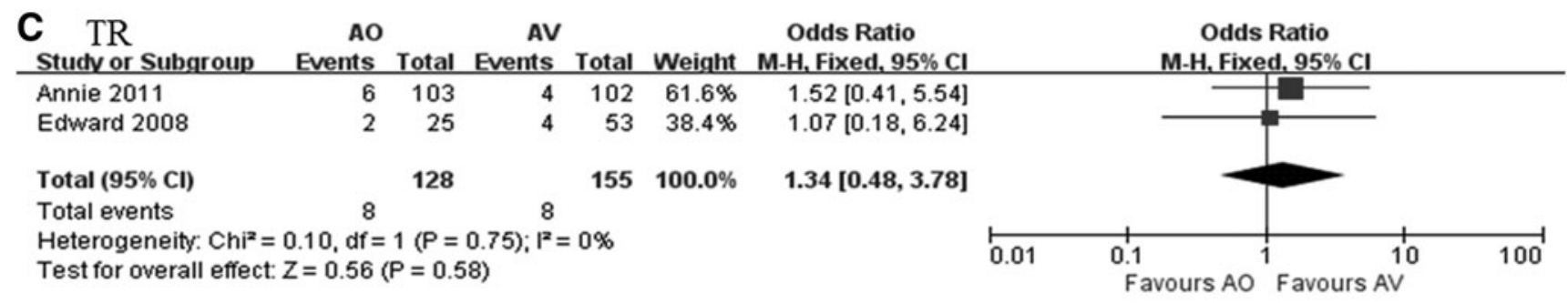

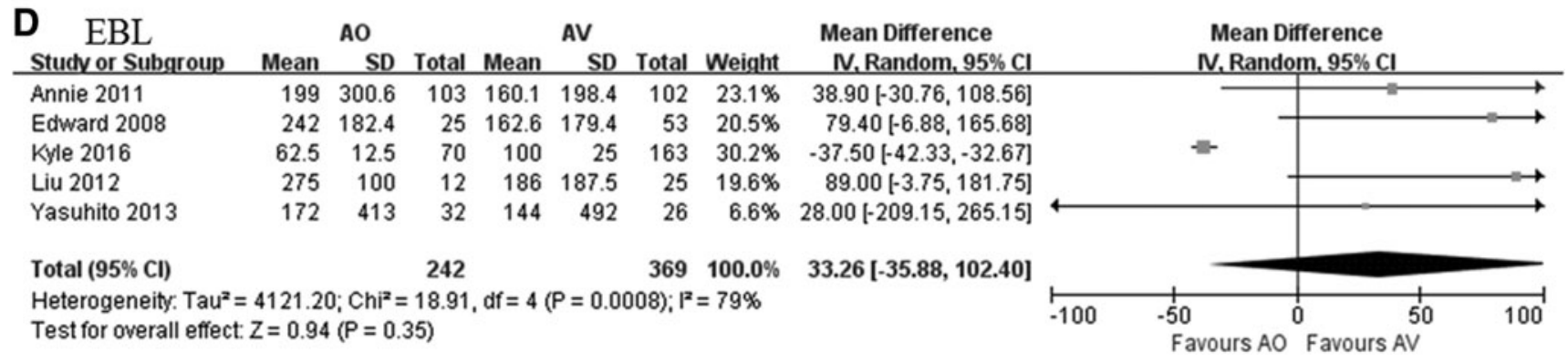

FIG. 3. Forest plots of surgical outcomes. (A) WI: (B) OT: (C) TR: (D) EBL.

after AO and AV clamping in a solitary kidney porcine model. They found that clamping of the artery alone during open surgery had better effect on protecting the kidney function from WI compared to AV occlusion. However, this benefit was not found during laparoscopic porcine surgery, perhaps because of pneumoperitoneum limited renal vein backflow, which might provide some level of oxygen for renal parenchyma. ${ }^{4}$

On the contrary, the results in human studies which had more complicated clinical settings were not consistent with animal studies. Gong and coworkers conducted a retrospective study and concluded that $\mathrm{AO}$ clamping might provide superior renal preservation during LPN. ${ }^{8}$ Some other studies reached different conclusions under more complicated clinical settings. Funahashi et al. reported that ischemic renal injury of AO clamping during LPN was smaller compared with AV clamping when the ischemic time was prolonged to 25 minutes. $^{2}$ Liu et al. assessed renal oxygenation profiles during PN using digital light processing-hyperspectral imaging and found that there was also no significant retrograde venous oxygen delivery and renal functional advantage for AO clamping in patients of difficult $\mathrm{PN}$ where prolonged ischemia time might have mitigated any benefits. ${ }^{12}$ Blum et al. and Imbeault and coworkers had similar conclusions and believed that AV and AO techniques were associated with similar renal function recovery in patients of LPN. ${ }^{6,7}$

Conventionally, the renal function recovery after PN was based on assessments of the serum creatinine level, eGFR, or percentage change of eGFR. However, these parameters evaluated the overall renal function, without considering the compensation by the functioning of contralateral kidney. Therefore, quantification of split renal function is preferable for the precise evaluation of renal functional changes. Imbeault and coworkers used renal mercaptoacetyltriglycineLasix scintigraphy to assess the split renal function. ${ }^{6}$ Furthermore, the volume of resected nephron and ischemic 


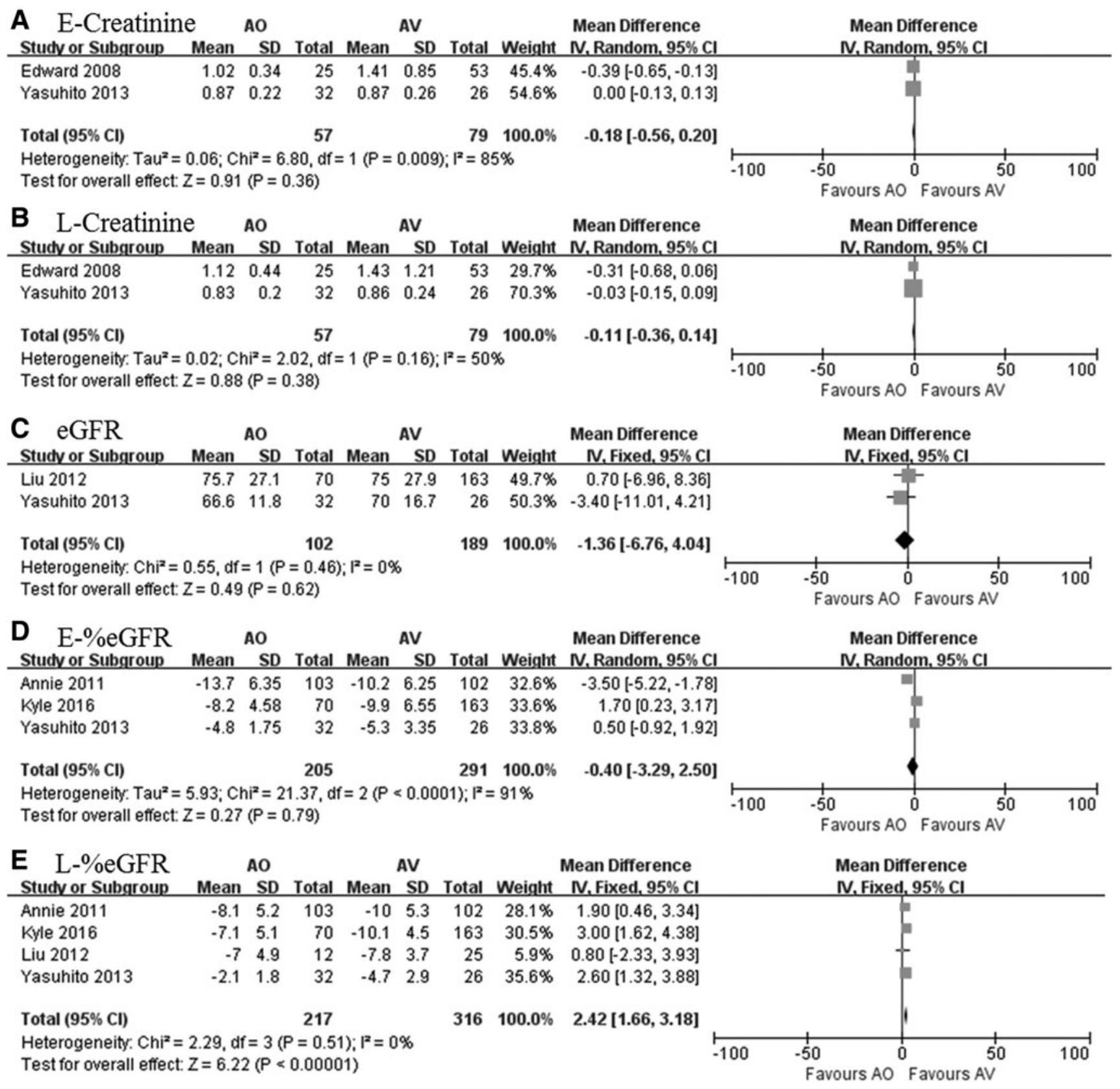

FIG. 4. Forest plots of functional outcomes. (A) E-Creatinine: (B) L-Creatinine: (C) eGFR: (D) E-\%eGFR: (E) L-\%eGFR.

injury in the preserved renal tissue was associated with renal function after PN, and conventional parameters cannot reflect these factors. A more specific method was applied in Funahashi et al.'s study. They assessed effective renal plasma flow by regional ${ }^{99 \mathrm{~m}}$ Technetium-mercaptoacetyltriglycine $\left({ }^{99 \mathrm{~m}} \mathrm{Tc}-\mathrm{MAG} 3\right)$ uptake, which could assess only of the ischemic damage to the surgically preserved renal tissue after PN without being affected by tumor or patient characteristics. $^{2}$ In addition, renal oxygenation was used as a new parameter to measure instant renal function, and percent renal parenchymal oxyhemoglobin $\left(\% \mathrm{HbO}_{2}\right)$ during $\mathrm{AO}$ vs $\mathrm{AV}$ occlusion was evaluated by DLP-HSI. The results showed that $\% \mathrm{HbO}_{2}$ profiles during $\mathrm{PN}$ were similar for $\mathrm{AO} v s \mathrm{AV}$ clamping, although in the $\mathrm{AV}$ group, but not the $\mathrm{AO}$ group, a lower mean ischemic $\% \mathrm{HbO}_{2}$ was associated with lower eGFR at the last follow-up and remained significant on multivariable analysis. ${ }^{12}$

Although the relationship between these two clamping techniques on renal function recovery is not clear, it seems that WI is a strong predictor of decrease in renal function. In an animal model, the $\% \mathrm{HbO}_{2}$ of $\mathrm{AO}$ clamping were significantly higher than AV clamping between 16 and 24 minutes after vascular occlusion, and it became similar after $\sim 36$ minutes, indicating a possible "ischemic window" in which AO occlusion may provide benefit over AV occlusion. ${ }^{13} \mathrm{Si}-$ milarly, in a human study by Funahashi et al., they used regional ${ }^{99 \mathrm{~m}} \mathrm{Tc}-\mathrm{MAG} 3$ uptake to assess the operated kidney and found that longer WI was correlated with more decrease 
in renal function in both groups, but this decrease was significantly less in the $\mathrm{AO}$ group when the ischemic time was more than 25 minutes. $^{2}$ In another study by Liu et al., the value of $\% \mathrm{HbO}_{2}$ quickly reached an "ischemic plateau" in both groups after vascular occlusion time within 45 minutes, with no significant difference between the groups; however, when the vascular clamping time went beyond 45 minutes, the $\% \mathrm{HbO}_{2}$ in both groups dropped sharply from plateau levels, indicating a possible "ischemic window" as well. ${ }^{12}$ As a result, the vascular occlusion time during LPN should not be longer than 45 minutes, no matter which clamping technique was applied.

The present meta-analysis included two retrospective and three prospective studies, including 242 patients who underwent AO clamping and 369 patients who underwent AV clamping, which compared $\mathrm{AO}$ and $\mathrm{AV}$ clamping in $\mathrm{PN}$ for RCC. The analysis consists of three main parts: meta-analysis of clinical characteristics, surgical outcomes, and functional outcomes. The first part has shown that patients in the AO clamping group had significantly lower RENAL Score (WMD: 0.36, $p=0.007$ ) and have similar BMI and TS. The complexity of renal masses in AV clamping group was higher than AO clamping group and the reason may be that $\mathrm{AV}$ clamping which blocked venous backflow could provide clearer visualization of the renal tumor margins during operations. The analysis of surgical outcomes showed similar WI, OT, TR, and EBL between two groups. These results suggested that AO clamping would not increase the difficulty of operation, which was commonly thought to be caused by venous backflow bleeding, and that AV clamping could not shorten the WI time to preserve more renal function. Finally, in the functional assessment, when the renal function was valued by creatinine, the two techniques were equivalent both at short and long term and no significant differences were detected in the eGFR variations and the early postoperative percentage change of eGFR as well. Interestingly, the AV clamping technique had a significant higher postoperative percentage change of eGFR decrease at last follow-up (WMD: 2.42, $p<0.00001$ ). When the renal function was estimated by postoperative percentage change of eGFR decrease, which was more accurate than creatinine and eGFR, the results proposed that $\mathrm{AO}$ clamping might produce less ischemic damage in the long term compared to clamping renal artery and vein together.

The present study has the following limitations that should be taken into consideration. First, no prospective RCT studies were included, indicating potential risk of bias of our findings. Second, obvious heterogeneity was identified in some analyses possibly because of the differences in parameters, such as study design, follow-up duration, and the patients' baseline characteristics, in the included studies. Third, the assessment parameters of renal function are not the same and not referred to as split renal function. Finally, oncologic outcomes are not observed between these two groups in all selected studies.

\section{Conclusion}

AO clamping would not increase the difficulty of LPN for the similar surgical results between $\mathrm{AO}$ and AV clamping. Although there is no significant difference in renal function preservation in short term after operations, AO clamping might produce less ischemic damage in the long term. These results suggest that $\mathrm{AO}$ clamping might be a better choice for LPN in long term. RCT studies with larger sample numbers, and long-term follow-up and split renal function assessment should be conducted in the future to confirm our conclusion.

\section{Acknowledgment}

We thank all the patients participated in this study.

\section{Author Disclosure Statement}

No competing financial interests exist.

\section{Funding Information}

No funding was received for this article.

\section{Supplementary Material}

Supplementary Figure S1

Supplementary Figure S2

\section{References}

1. Klatte T, Vincenzo Ficarra, Christian Gratzke, et al. A literature review of renal surgical anatomy and surgical strategies for partial nephrectomy. Eur Urol 2015;68:980-992.

2. Funahashi Y, Kato M, Yoshino Y, Fujita T, Sassa N, Gotoh M. Comparison of renal ischemic damage during laparoscopic partial nephrectomy with artery-vein and artery-only clamping. J Endourol 2013;28:306-311.

3. Colli JL, Wang Z, Johnsen N, Grossman L, Lee BR. Clamping renal artery alone produces less ischemic damage compared to clamping renal artery and vein together in two animal models: Near-infrared tissue oximetry and quantitation of 8-isoprostane levels. Int Urol Nephrol 2013;45: 421-428.

4. Orvieto MA, Zorn KC, Mendiola F, Lyon MB, Mikhail AA, Gofrit ON, Shalhav AL. Recovery of renal function after complete renal hilar versus artery alone clamping during open and laparoscopic surgery. J Urol 2007; 177:2371-2374.

5. McLoughlin GA, Heal MR, Tyrell IM. An evaluation of techniques used for the production of temporary renal ischaemia. Br J Urol 1978;50:371-375.

6. Imbeault A, Pouliot F, Finley DS, Shuch B, Dujardin T. Prospective study comparing two techniques of renal clamping in laparoscopic partial nephrectomy: Impact on perioperative parameters. J Endourol 2012;26:509-514.

7. Blum KA, Paulucci DJ, Abaza R, et al. Main renal artery clamping with or without renal vein clamping during robotic partial nephrectomy for clinical T1 renal masses: Perioperative and long-term functional outcomes. Urology 2016;97:118-123.

8. Gong EM, Zorn KC, Orvieto MA, Lucioni A, Msezane LP, Shalhav AL. Artery-only occlusion may provide superior renal preservation during laparoscopic partial nephrectomy. Urology 2008;72:843-846.

9. Moher D, Liberati A, Tetzlaff J, Altman DG. Preferred reporting items for systematic reviews and meta-analyses: The PRISMA statement. J Clin Epidemiol 2009;62:1006-1012.

10. Stroup DF, Berlin JA, Morton SC, et al. Meta-analysis of observational studies in epidemiology: A proposal for reporting. JAMA 2000;283:2008-2012.

11. Zeng X, Zhang Y, Kwong JS, Zhang C, Li S, Sun F, Niu Y, $\mathrm{Du}$ L. The methodological quality assessment tools for 
preclinical and clinical studies, systematic review and meta-analysis, and clinical practice guideline: A systematic review. J Evid Based Med 2015;8:2-10.

12. Liu Z-W, Faddegon S, Olweny EO, Best SL, Jackson N, Raj GV, Zuzak KJ, Cadeddu JA. Renal oxygenation during partial nephrectomy: A comparison between artery-only occlusion versus artery and vein occlusion. J Endourol 2013;27:470-474.

13. Tracy CR, Terrell JD, Francis RP, et al. First Prize: Characterization of renal ischemia using DLP ${ }^{\circledR}$ hyperspectral imaging: A pilot study comparing artery-only occlusion versus artery and vein occlusion. J Endourol 2010;24:321325 .

Address correspondence to:

Yu Xie, $M D, P h D$

Department of Urology

Hunan Cancer Hospital and The Affiliated Cancer Hospital of Xiangya School of Medicine

Central South University 283 TongZiPo Road

Changsha 410006

Yuelu District

Hunan

China

E-mail:9789691@qq.com

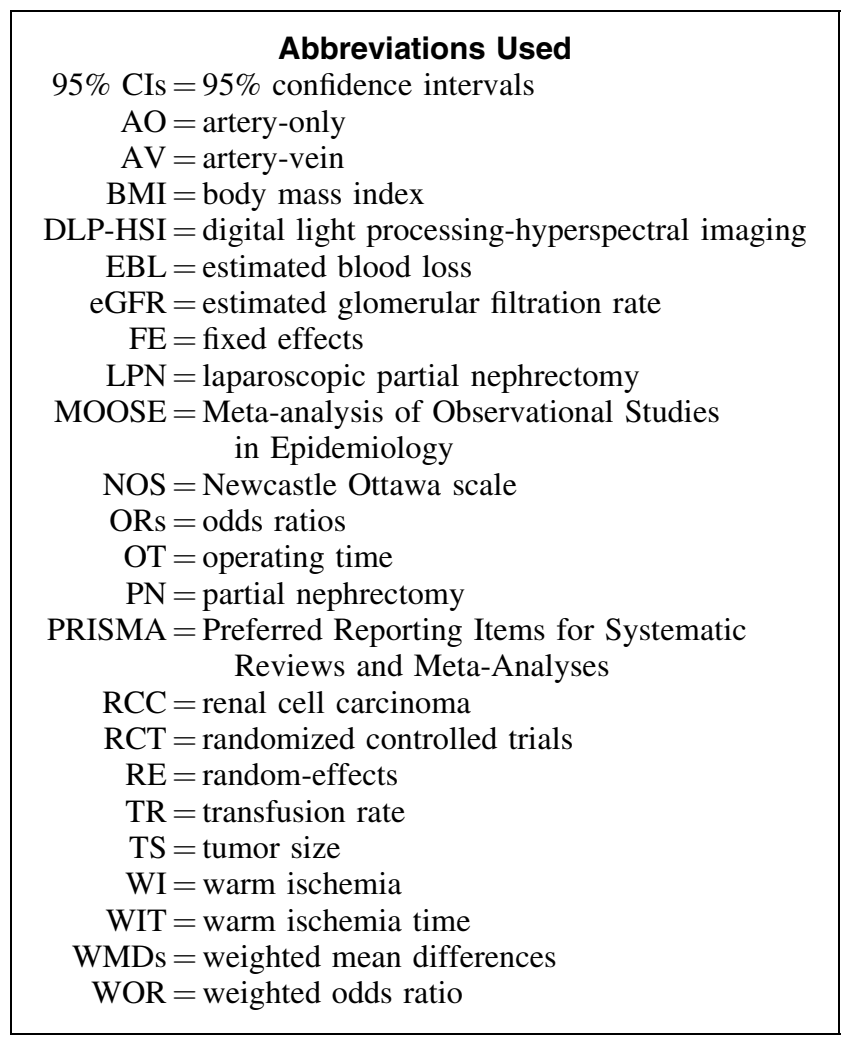

